\title{
Enhancing the Cooperative Transport of Multiple Objects
}

\author{
Antoine Decugnière ${ }^{1}$, Benjamin Poulain ${ }^{1}$, Alexandre Campo $^{1}$, Carlo Pinciroli ${ }^{1}$, \\ Bruno Tartini $^{2}$, Michel Osée ${ }^{2}$, Marco Dorigo ${ }^{1}$, and Mauro Birattari ${ }^{1}$ \\ ${ }^{1}$ IRIDIA, CoDE, Université Libre de Bruxelles, Brussels, Belgium \\ \{adecugni, bpoulain, acampo, cpinciro, mdorigo, mbiro\}@ulb.ac.be \\ 2 BEAMS, Université Libre de Bruxelles, Brussels, Belgium \\ $\{$ btartini, mosee\}@ulb.ac. be
}

\begin{abstract}
In this paper we present an approach to the cooperative transport of multiple objects in swarm robotics. The approach is motivated by the observation that the performance of cooperative transport in insect colonies as well as in groups of robots grows in a super linear way with the number of individuals participating in the transport. The transport relies on a cart in which multiple objects are collected and stored before being moved to destination. The cart is carried by a group of robot that would be otherwise allocated to the transport of single objects. The cart is endowed with computational and communication abilities that allow it to cooperate with the transporting robots. This research is carried out within the framework of the Swarmanoid project and aims at enhancing the transport capabilities of the robot swarms developed in this project.
\end{abstract}

\section{Introduction}

Cooperative transport is a classical problem studied in the collective robotics literature [12/3/4/5]. Robots have to perform cooperative transport when a task requires the transport of an object that a single robot is not able to handle and move. Approaches to solve this problem have typically taken inspiration from biological systems, especially from colonies of social insects [6/788.

In particular, ant colonies display two different transport behaviours: solitary transport and group transport. In some ant species, group transport is observed when a prey is larger or heavier than the transporting capability of a single ant. In this case, ants have the option either to cut the prey in pieces and to transport each piece individually, or to cooperate to transport the whole prey as a group. Studies show that transporting the prey as a whole in group is usually more efficient than the solitary transport of pieces $[9[10]$.

Kube and Zhang developed a model of cooperative transport inspired by the behaviour of socials insects 7 . This model was then demonstrated by Kube and Bonabeau with experiments involving a group of real robots [11. Additional experiments have shown that transport performance grows in a super linear way with the number of robots, which is similar to what is observed with ants [12]13. 
In this paper, we present an approach to enhance the ability of a group of robots to transport objects cooperatively. The objects to be transported are gathered in a cart and are then transported collectively as a single entity. The research presented in this paper is carried out within the framework of the Swarmanoid project [14, a Future and Emerging Technologies (FET-OPEN) project funded by the European Commission. The goal of the project is to develop a swarmanoid, that is, a swarm-based alternative to a humanoid robot. In a swarmanoid, the functionalities that one typically expects in a humanoid robot are distributed among the individuals of an heterogeneous swarm of robots. The swarmanoid is composed of (i) a number of eye-bots, flying robots that are able to explore a scene and to localize objects; (ii) a number of hand-bots, manipulators that are able to climb and to grasp object; and (iii) a number of foot-bots, robot rovers that are able to move on the ground and to transport objects or other robots. In the spirit of the Swarmanoid project, we design a fourth kind of robot: the cart-bot. A cart-bot is able to store a number of objects, to cooperate with hand-bots in order to facilitate the loading and unloading of objects, and to cooperate with foot-bots to ease its own transport.

The rest of the paper is organized as follows: in Section 2 we detail the integration of the cart-bot in the Swarmanoid project. In Section 3 we describe the final design of the cart-bot hardware. Finally, in Section 4 we conclude the paper.

\section{Integration of the Cart-Bot in the Swarmanoid Project}

The Swarmanoid project is developed around a main scenario, which illustrates the use of morphologically specialised robots to solve a complex mission in a human-like environment. In the following, we describe how the cart-bot can be used to enhance transport tasks within the scope of this scenario.

In the Swarmanoid scenario, an heterogeneous group of robots is employed to locate books situated on shelves, collect them, and bring them to a target place. For practical reasons, rather than with real books, robots deal instead with models of books that we call book-bots. A book-bot is made of foam so that it causes less damages if it inadvertently falls on the ground or on a robot; moreover, it features a number of LEDs on the spine to facilitate its localisation.

A typical unfolding of the scenario is as follows: first the eye-bots explore the environment. Once they have located a number of book-bots, they guide other robots to them. The hand-bots that have extended manipulation capabilities are transported by the foot-bots close to these book-bots. The hand-bots get a hold of the book-bots one by one. In a scenario without the cart-bot, each book-bot is transported by a system composed of a hand-bot holding a book-bot and a number of foot-bots carrying the hand-bot.

With the cart-bot included in the scenario, once a hand-bot grasps a bookbot, it lays it on the depository area of the cart-bot, as shown in Figure 1(a). The cart-bot automatically swallows the book-bot to store it inside his rack and the hand-bot is therefore free to pursue the collection of another book-bot. As soon as the cart-bot is full or has loaded the required number of book-bots, it advertises to surrounding foot-bots that it is ready to be transported. Once 


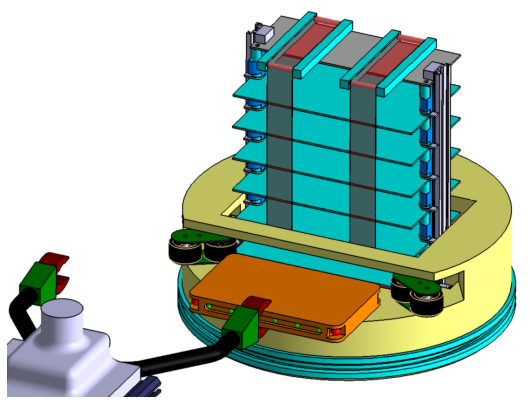

(a)

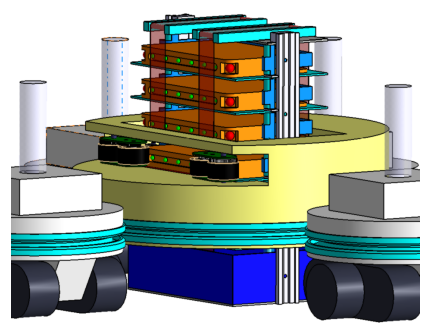

(c)

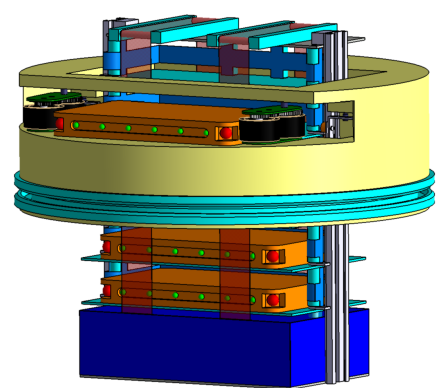

(b)

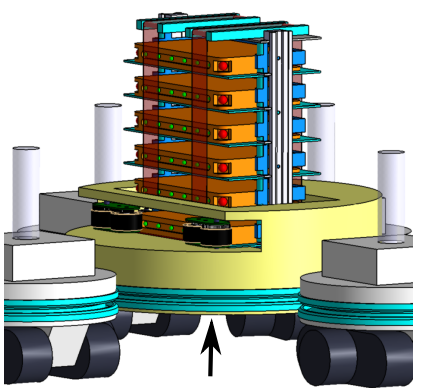

(d)

Fig. 1. (a) Book-bot put on the loading bay by a hand-bot. (b) Base elevation on the rack to reach a high slot. (c) Foot-bots docked to the cart-bot. (d) Rack elevated to remove friction with the ground.

foot-bots are physically connected to the cart-bot, it lifts up the part of its body that was previously in contact with the floor- see Figures 1(c) and 1(d). By doing this, the cart-bot prevents any friction with the ground, therefore facilitating its transport. Eventually, the cart-bot communicates with the connected foot-bots to let them know that transport can be performed at any time.

The integration of the cart-bot in Swarmanoid allows to study stacked transport against single transport of multiple objects. Furthermore, contrary to the hand-bot, the cart-bot is specialized for transport: it is able to store a number of book-bots, it has no fragile external components like manipulators do, book-bots are stabilized inside the cart-bot and may not fall on the floor. Lastly, the cartbot is large and foot-bots can connect to it all around such that the resulting assembly is very stable.

\section{Hardware Design}

The two main functionalities of the cart-bot are storing book-bots in a single rack and allowing the single cooperative transport of all the gathered book-bots by the foot-bots. The design of these functionalities is described in the following. 


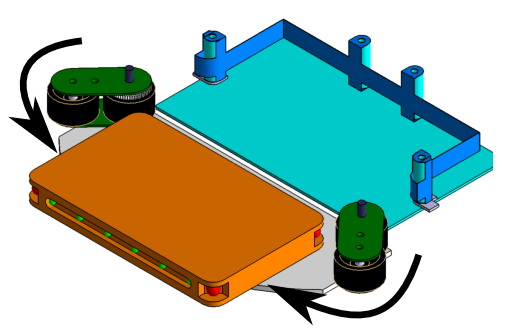

(a)

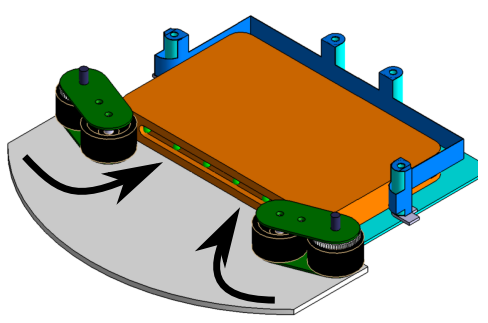

(c)

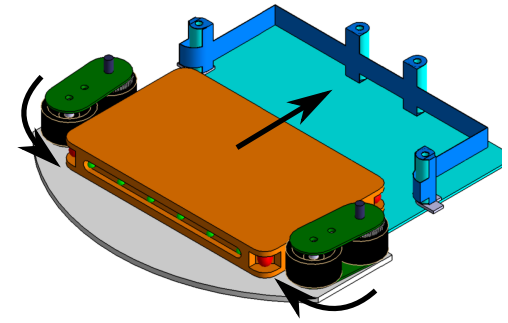

(b)

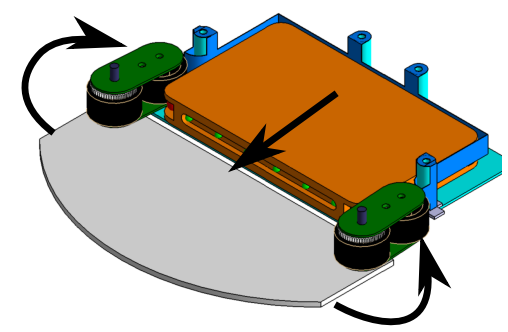

(d)

Fig. 2. (a) The arms rotating towards a book-bot on the loading bay. (b) The wheels pushing the book-bot inside a slot. (c) The arms finishing to push the book-bot in the slot. (d) The arms reversed to get a book-bot from a slot.

\subsection{Storing Ability}

In order to store the book-bots, the cart-bot introduces them inside the slots of a storing system called the rack. This can be done in two different ways: either by having the hand-bot inserting a book-bot directly in a selected slot, or by having the hand-bot laying a book-bot down on a specific bay and then moving the bookbot to the appropriate slot through some loading and unloading mechanisms. The second approach turns out to be simpler to implement and requires less accuracy in the positioning of the book-bot by the hand-bots.

In the design we developed, the loading and the unloading functions are carried out by a single mechanism. This loading/unloading mechanism consists of two rotating arms with wheels at their ends. The arms are located on both sides of the loading bay, right in front of the slots as it can be seen in Figures 2(a), 2(b), 2(c) and $2(\mathrm{~d})$, By rotating in one direction or in the opposite, these arms can reach a bookbot either when the book-bot is on the loading bay - as shown in Figure 2(a) or when the book-bot is inside the slot - as shown in Figure 2(d). In particular, Figure 2(b) shows how the arms, pressed against a book-bot, push the book-bot itself inside the slot. The wheels turn and push the book-bot in the same direction. This always ensures a tight contact and a good grip with the book-bot. Once the book-bot has been swallowed by the wheels, the arms complete their rotation and push the book-bot inside the slot to their final storage position - as shown in Figure 2(c). 


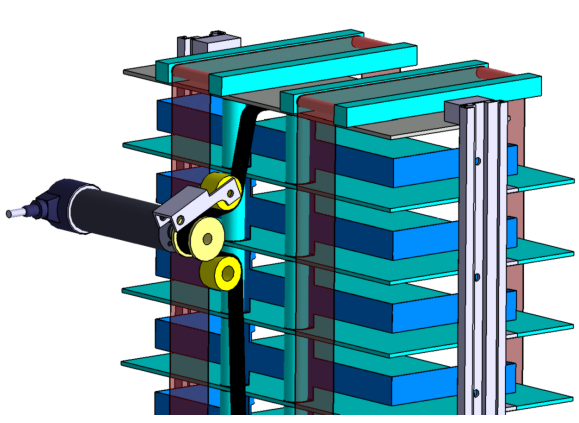

(a)

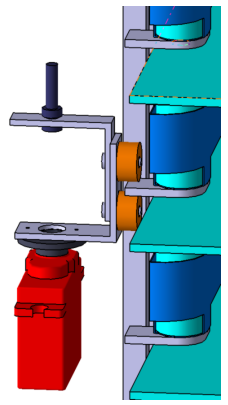

(b)

Fig. 3. (a) Belt transmission and linear guides. (b) Brake mechanism on a slot.

During the loading phase, the trajectory followed by the book-bot is determined by the angular position of the arms. In particular, in order to insure that the book-bot enters the slot with a correct angle, the rotating arms must assume a symmetric angular position. During the whole procedure, the speed and the direction of the motors are adapted in order to correct the trajectory of the bookbot. If an incoming book-bot gets stuck, the failure is detected by a current peak on the blocked motor or by the incomplete angular positioning of the rotating arms. The motors are reversed and the whole procedure is repeated.

The slots where the book-bots are stored are engineered in a way that they form a vertical rack of horizontal slots - see Figure 1(a). The storing system manages the slots and takes care to align the loading bay and the loading/unloading system with the slot in which the book-bot has to be inserted. In order to achieve correct alignment, the rack can be moved relatively to the base platform or the base platform can be moved relatively to the rack, depending on whether the cart-bot is being held by foot-bots or it lies on the ground - see Figure 1(b). This allows the system to lift up the rack and ease transport by preventing any friction with the ground, as described in Section 3.2

The structure of this system is very similar to an elevator architecture and can be controlled through a classical elevator-like regulation. It uses linear guides and a timing (toothed) belt - see Figure 3(a), The belt is driven by a pulley fixed directly on the shaft of a motor with an encoder. The belt transmission was preferred to a screw drive transmission because of weight and efficiency concerns, but it has the drawback of not being auto-blocking. This led us to implement a braking system that insures precise alignment of the slot to the loading bay. The braking system is designed to be finely adjustable in height and is based on simple and lightweight components - see Figure 3(b).

The presence of a book-bot in a slot is perceived as the success of the loading and is memorized by the storing system. To ensure a reliable perception of the status of the slots, we introduce redundancy with optical sensors. This information is critical to know where are the free slots and when the cart-bot is ready to be transported. 


\subsection{Transportability}

To display its status, for example when it is ready to be transported, the cart-bot uses a ring of RGB LEDs. This color ring can also be used coordinate the footbots by indicating them in what position they should dock. Docking is achieved by the foot-bots by gripping a docking ring on the cart-bot. This docking ring is positioned on the lowest part of the base platform. The cart-bot uses the mechanism of the storing system to elevate the ring up to the height of the gripper of the foot-bots. This prevents the ring to be in the way of a book-bot brought horizontally by a hand-bot to the loading bay when the base platform is lowered-see Figure 1(a).

During all these manipulations the rack of slots is resting on the floor. It presents high resistance to the foot-bots trying to push or pull it. Docked footbots may rely on this resistance to decide to recruit more foot-bots, as exposed in [15]. Once enough foot-bots are docked, the rack is lifted up so that it doesn't touch the floor anymore and the friction to the ground is canceled-see Figure 1(c). Thanks to this mechanism, the cart-bot has a good ground clearance when transported. The system formed by the cart-bot and the foot-bots is very stable with a large base and has a good mobility.

\section{Conclusions}

In the paper we have discussed the problem of the cooperative transport of multiple objects by a swarm of robots. In the approach we adopted, rather than transporting objects one by one, these are gathered in a cart which is subsequently transported by the swarm. With this approach, the transport performance grows super linearly with the size of the swarm, with the classic limitations induced by the problem of coordinating the movement of the swarm [12]. In the paper, we have analyzed the problem of transporting multiple objects within the framework of the Swarmanoid project. In particular, we have described the features and the design of the cart-bot, a robot which is able to store objects, the bookbots, and ease the task of other robots, such as the foot-bots that are intended to carry out transport.

The cart-bot enhances the robustness provided by the distributed hardware and control [16] in the transport of book-bots. The cart-bot secures the stored book-bots to avoid losing them during the transport due to collisions or to the roughness of the terrain. The large round shape of the cart-bot allows the footbots to dock all around it: this results in a very stable assembly. Moreover, the transporting assembly is not much sensitive to terrain condition, thanks to a good ground clearance obtained through the elevation of the rack of the cart-bot after the docking with the foot-bots.

To ensure a robust transport, the cart-bot is designed to be simple and reliable. During transport, the cart-bot can be seen as a single resistant entity, as it has no fragile external parts. The use of the same mechanisms for different functionalities minimizes the complexity and reduces the global weight of the cart-bot: rotating arms are used for loading and unloading and an elevation 
system is used for reaching different slots of the rack when loading and unloading and for elevating the rack itself during the transport. Finally, the cart-bot is mainly built with generic electronic and mechanical components, which makes the cart-bot easy to maintain.

Acknowledgments. The authors thank Francesco Mondada and Michael Bonani for the useful discussions and the valuable advice. The research described in the paper was carried out in the framework of Swarmanoid, a project funded by the Future and Emerging Technologies programme (IST-FET) of the European Commission under grant IST-022888. The work was partially supported by the project ANTS, an Action de Recherche Concertée funded by the Scientific Research Directorate of Belgium's French Community. Alexandre Campo, Marco Dorigo, and Mauro Birattari acknowledge support from the fund for scientific research F.R.S.-FNRS of Belgium's French Community.

\section{References}

1. Matarić, M., Nilsson, M., Simsarian, K.: Cooperative multi-robot box-pushing. In: Proceedings of the IEEE/RSJ International Conference on Intelligent Robots and Systems, Pittsburg, PA, pp. 556-561. IEEE Computer Society Press, Los Alamitos (1995)

2. Pereira, G.A.S., Kumar, V., Spletzer, J., Taylor, C.J., Campos, M.F.M.: Cooperative transport of planar objects by multiple mobile robots using object closure. In: Proceedings of the 8th International Symposium on Experimental Robotics, ISER 2002, Sant'Angelo d'Ischia, Italy (2002)

3. Wang, Z., Kumar, V.: Object closure and manipulation by multiple cooperative mobile robots. In: Proceedings of the IEEE International Conference on Robotics and Automation, ICRA2002, Washington, DC, pp. 394-399. IEEE Computer Society Press, Los Alamitos (2002)

4. Groß, R., Dorigo, M.: Cooperative transport of objects of different shapes and sizes. In: Dorigo, M., Birattari, M., Blum, C., Gambardella, L.M., Mondada, F., Stützle, T. (eds.) ANTS 2004. LNCS, vol. 3172, pp. 107-118. Springer, Heidelberg (2004)

5. Campo, A., Nouyan, S., Birattari, M., Groß, R., Dorigo, M.: Negotiation of goal direction for cooperative transport. In: Dorigo, M., Gambardella, L.M., Birattari, M., Martinoli, A., Poli, R., Stützle, T. (eds.) ANTS 2006. LNCS, vol. 4150, pp. 191-202. Springer, Heidelberg (2006)

6. Bonabeau, E., Dorigo, M., Theraulaz, G.: Swarm Intelligence: From Natural to Artificial Systems. Oxford University Press, New York (1999)

7. Kube, R.C., Zhang, H.: Collective robotics: from social insects to robots. Adaptive Behaviour 2(2), 189-218 (1994)

8. Martinoli, A., Mondada, F.: Collective and cooperative group behaviours: Biologically inspired experiments in robotics. In: Proceedings of the Fourth Symposium on Experimental Robotics, ISER-1995, Stanford, California, USA (June 1995)

9. Moffett, M.W.: Cooperative food transport by an asiatic ant. National Geographic Research 4, 386-394 (1988)

10. Traniello, J.F.A., Beshers, S.N.: Maximization of foraging efficiency and ressource defense by group retrieval in the ant Formica schaufussi. Behavioral Ecology and Sociobiology 29, 283-289 (1991) 
11. Kube, C., Bonabeau, E.: Cooperative transport by ants and robots. Robotics and Autonomous Systems 30(1 - 2), 85-101 (2000)

12. Mondada, F., Bonani, M., Guignard, A., Magnenat, S., Studer, C., Floreano, D.: Superlinear physical performances in a swarm-bot. In: Capcarrère, M.S., Freitas, A.A., Bentley, P.J., Johnson, C.G., Timmis, J. (eds.) ECAL 2005. LNCS (LNAI), vol. 3630, pp. 282-291. Springer, Heidelberg (2005)

13. O’Grady, R., Groß, R., Christensen, A., Mondada, F.M., Bonani, M.D.: Performance benefits of self-assembly in a swarm-bot. In: Proceedings of the 2007 IEEE/RSJ Intl. Conference on Intelligent Robots and Systems, IROS 2007, San Diego, CA (2007)

14. Dorigo, M., Tuci, E., Groß, R., Trianni, V., Labella, T., Nouyan, S., Ampatzis, C., Deneubourg, J.L., Baldassarre, G., Nolfi, S., Mondada, F., Floreano, D., Gambardella, L.: The SWARM-BOTS project. In: Şahin, E., Spears, W.M. (eds.) Swarm Robotics 2004. LNCS, vol. 3342, pp. 31-44. Springer, Heidelberg (2005)

15. Groß, R.: Self-assembling robots. PhD thesis, Université Libre de Bruxelles, Brussels, Belgium (2007)

16. Mondada, F., Pettinaro, G.C., Guignard, A., Kwee, I., Floreano, D., Deneubourg, J.L., Nolfi, S., Gambardella, L., Dorigo, M.: Swarm-bot: a new distributed robotic concept. Autonomous Robots 17(2-3), 193-221 (2004) 